\title{
The Effectiveness of Moxibustion: An Overview During 10 Years
}

\author{
Song-Yi Kim, ${ }^{1,2}$ Younbyoung Chae, ${ }^{1,2}$ Seung Min Lee, ${ }^{1}$ Hyejung Lee, ${ }^{1,2}$ and Hi-Joon Park ${ }^{1,2}$ \\ ${ }^{1}$ Department of Meridian and Acupoint, College of Korean Medicine, Kyung Hee University, 1 Hoegidong, Dongdaemungu, \\ Seoul, 130-701, Republic of Korea \\ ${ }^{2}$ Acupuncture and Meridian Science Research Center (AMSRC), Kyung Hee University, 1 Hoegidong, Dongdaemungu, \\ Seoul, 130-701, Republic of Korea \\ Correspondence should be addressed to Hi-Joon Park, acufind@khu.ac.kr
}

Received 21 May 2009; Accepted 30 August 2009

Copyright () 2011 Song-Yi Kim et al. This is an open access article distributed under the Creative Commons Attribution License, which permits unrestricted use, distribution, and reproduction in any medium, provided the original work is properly cited.

\begin{abstract}
Moxibustion has been used to treat various types of disease. However, there is still insufficient evidence regarding its effectiveness. This study was performed to summarize and evaluate the effectiveness of moxibustion. A search was performed for all randomized controlled trials in PubMed between January 1998 and July 2008 with no language restriction. The results yielded 47 trials in which six moxibustion types were applied to 36 diseases ranging from breech presentation to digestive disorders. Moxibustion was compared to three types of control group: general care, Oriental medical therapies or waiting list. Moxibustion was superior to the control in 14 out of 54 control groups in 46 studies. There were no significant differences among groups in 7 studies, and the outcome direction was not determined in 33 studies. Seven studies were included in a meta-analysis. Moxibustion was more effective than medication in two ulcerative colitis studies (relative risk (95\% CI), $\left.2.20(1.37,3.52), P=.001, I^{2}=0 \%\right)$. Overall, our results did not support the effectiveness of moxibustion in specific diseases due to the limited number and low quality of the studies and inadequate use of controls. In order to provide appropriate evidence regarding the effectiveness of moxibustion, more rigorous clinical trials using appropriate controls are warranted.
\end{abstract}

\section{Introduction}

Acupuncture and moxibustion, representative therapeutic modalities in traditional medicine for more than 2500 years [1], are still being used in primary healthcare systems in East Asia [2]. Traditional treatments including acupuncturerelated therapies (acupuncture, moxibustion and acupressure) and herbal remedies account for $40 \%$ of all healthcares in China [3]. In Korea, it was reported that $67 \%$ of Korean medical doctors use moxibustion as a therapeutic tool alone or in addition to their clinical practice $[4,5]$. Acupuncture and moxibustion are similar regarding stimulation of acupuncture points on the Meridian [6]. Moxibustion uses thermal and chemical stimulants by burning herbal materials, including mugwort (Artemisia vulgaris, moxa), whereas acupuncture uses physical stimulation via insertion of needles [7]. The therapeutic components of moxibustion are assumed that the combination of heat (burning pain and heat stress), tar (extract), aroma (fume) and psychological stress [8]. Among them, heat stimulation and chemical action of ignited moxa is the most important variable for moxibustion [9]. As the method of acupuncture and moxibustion is different, there are functional distinctions between them. Acupuncture generally makes body release heat, or eliminates pathogen while moxibustion mainly makes body warm and recruits healthy qi [10]. Therefore, acupuncture and moxibustion are used to cover different conditions, while they share some common applications at the same time [11]. Recently, pre-clinical studies suggested that moxibustion boosts the immune system $[8,12]$ and enhances physiological functions [13]. In addition, accumulating clinical data support the use of moxibustion [1419]. However, there have been few systematic reviews of the application of moxibustion, except in cases of breech presentation [20-22], and evidence regarding the efficacy of moxibustion is very limited.

The aims of this review are to summarize the present status of clinical research on moxibustion and to evaluate the evidence for the effectiveness of moxibustion.

\section{Methods}

2.1.Search Strategies and Selection of Studies. A search was performed in PubMed (http://www.ncbi.nlm.nih.gov/pubmed/), which offers better indexing and more search features [23], for the period spanning January 1998 to July 2008, with no 
language restriction. The literature search was performed using the key words "moxibustion" and "moxa", and reference lists from the original articles and reviews were examined for additional studies.

Studies fulfilling the following criteria were included: (i) studies presenting the results of a randomized controlled trial (RCT); and (ii) studies using moxibustion in the intervention group. Studies in which moxibustion were administered to both the intervention and control groups were excluded. Studies using warm needles (i.e., examining not the efficacy of moxibustion itself, but its effects in addition to acupuncture) were also excluded. No limitations were applied to the type of disease, outcome measure or control group.

2.2. Data Extraction and Quality Assessment. The data were extracted systematically in a pre-defined, standardized manner according to the study designs, number of subjects, intervention, and control groups. Selection, extraction, and quality assessment were performed independently by two reviewers (Kim SY and Lee S). Discrepancies were resolved by discussion between reviewers. A modified Jadad scale was used to assess the methodological quality of the included studies $[24,25]$. This scale assesses randomization (if the study was randomized, add 1 point; add an additional point for appropriate randomization and deduct 1 point for inappropriate randomization), patient and assessor blinding (add 1 point each), and reports of dropouts and withdrawals (add 1 point). The Jadad score ranges from 0 to 5 points. We considered trials with $\geq 3$ points to be of high quality.

2.3. Data Analysis. As all available studies related to the efficacy of moxibustion were included, the diseases for this review were very heterogeneous. Therefore, we categorized each disease according to the International Statistical Classification of Diseases and Related Health Problems, 10th Revision (ICD-10). All the outcomes included were reviewed. To interpret the results efficiently and briefly, with compensation for heterogeneity, two alternative analyses were used: the relative risk (RR) and classification score. For studies providing effective rates as an outcome measure, the $\mathrm{RR}$ and $95 \%$ confidence interval (CI) were calculated, and reanalyzed using the $\chi^{2}$ test. "Clinically cured" and "markedly effective" were included for success. When the RR could not be calculated, the appropriateness of outcomes related to the corresponding disease was discussed with experts from the Kyung Hee University Oriental Medical Hospital, followed by review of the statistical methods. For the classification score, the outcomes were defined as follows: (i) positive when moxibustion was significantly more effective than the control group (P); (ii) neutral when moxibustion was not significantly different from the control group (NEU); (iii) negative when the control group was significantly more effective than moxibustion (N); and (iv) not determined (ND) when the outcome measure was not appropriate for disease, the control was not adequate to prove the evidence of moxibustion, or the results were not clear. $\mathrm{P}$ and $\mathrm{N}$ were given only when the results of both author and reviewer were the same. If there was disagreement between authors and reviewers, the study was classified as ND. Meta-analyses were performed when the provided data were appropriate. The $I^{2}$ statistic describes the percentage of the total variability in study estimates that is due to heterogeneity rather than chance alone. The mean effect size was calculated using a random effects model, as we assumed that each study assessed different moxibustion treatments and thus different effects. Sensitivity analysis was performed using the $\chi^{2}$ test to examine the statistical significance of associations between trial methodological quality, country, languages, type of intervention, comparative control group and trial outcome based on classification score. SPSS software (Version 13.0) and Review Manager (RevMan 5.0; The Nordic Cochrane Centre, Copenhagen) were used for statistical analyses, and $P<.05$ was taken to indicate significance.

\section{Results}

3.1. Study Characteristics. A total of 737 potentially relevant studies were identified and screened for retrieval. Forty eight of these studies $[14,16,17,26-70]$ fulfilled the inclusion criteria for this review (Figure 1) and are presented in Table 1. Two papers by Liu et al. [27, 28] on malignant tumors described the same populations and were considered as one study. Therefore, we included 47 studies in this review. In total, moxibustion was compared to 54 control groups in 47 studies where seven of these were three-armed studies $[17,31,32,34,40,49,56]$.

Most of the studies were conducted in China (41 studies), three in Italy $[14,16,17]$, and three in Korea $[40,61,64]$. Thirty-three papers were written in Chinese, and the rest were in English.

3.2. Participants and Conditions. A total of 4434 patients (2274 in the moxibustion group, 2160 in the control group) participated in the studies, and the data from 4360 patients (2239 in the moxibustion group, 2121 in the control group) were analyzed. The average number of subjects in each group ranged from 5 to 130 in the moxibustion groups (mean \pm SD, $46.4 \pm 28.6)$ and 5 to 130 in the controls $(41.5 \pm 25.6)$. The median sample sizes per group (moxibustion and control) were 38 and 34, respectively.

The ICD-10 was used to categorize disorders treated with moxibustion. Thirty-six types of disease were included within 13 subcategories of the ICD-10. The most frequently examined disorders were diseases of the musculoskeletal system and connective tissue (Chapter XIII, $n=7$ ), the genitourinary system (Chapter XIV, $n=7$ ), pregnancy, childbirth and the puerperium (Chapter XV, $n=7$ ), and the digestive system (Chapter XI, $n=6)$. Others included diseases of the nervous system (Chapter VI, $n=4$ ), the respiratory system (Chapter $\mathrm{X}, n=4$ ), endocrine, nutritional and metabolic diseases (Chapter IV, $n=3$ ), neoplasms (Chapter II, $n=2$ ), diseases of the circulatory system (Chapter IX, $n=2$ ), the skin and subcutaneous tissues (Chapter XII, $n=2$ ), certain infectious and parasitic diseases (Chapter I, $n=1$ ), the blood and blood-forming organs and certain disorders involving the immune system (Chapter III, $n=1$ ), and the eye, adnexa, ear and mastoid process 


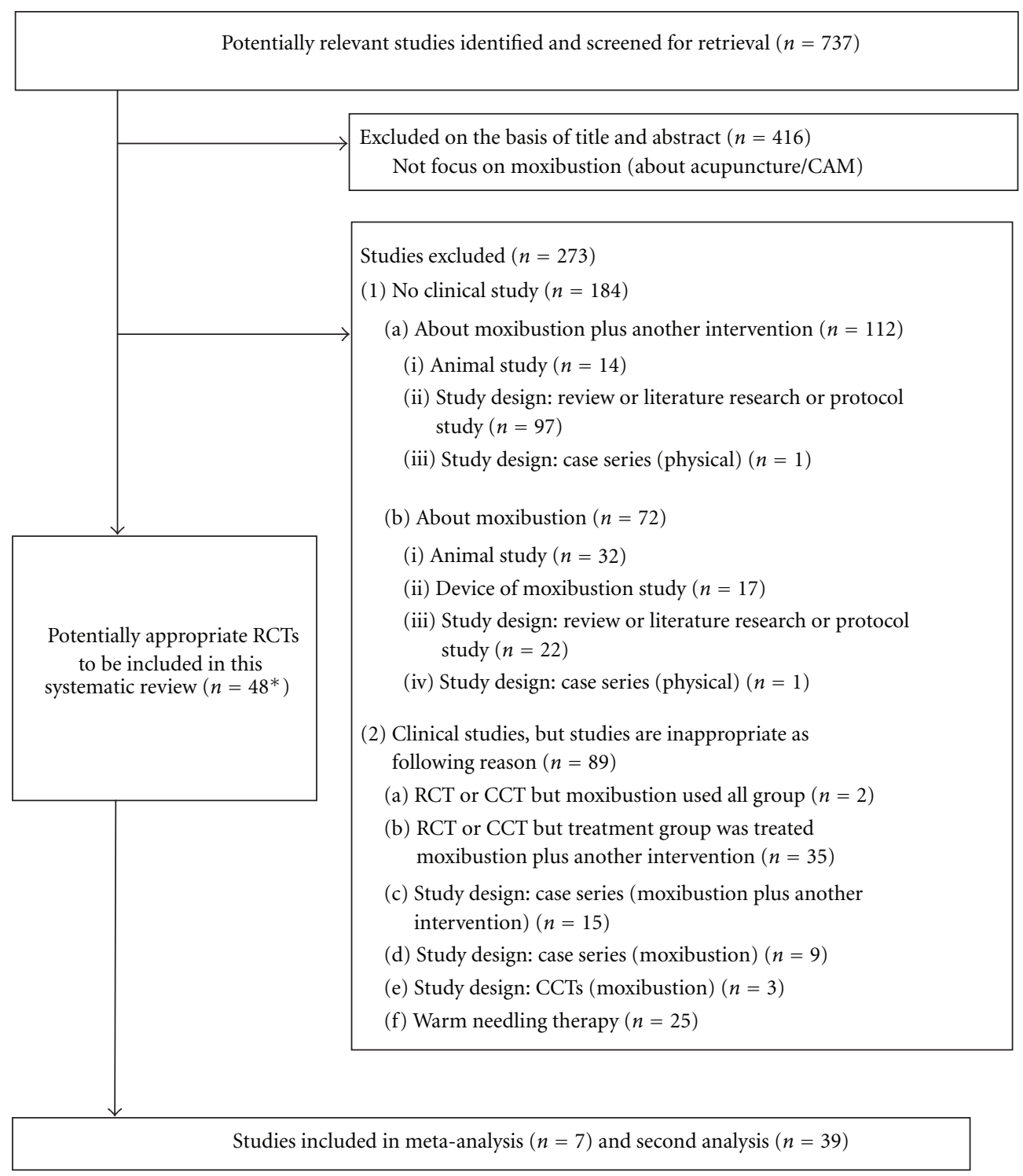

Figure 1: Flow diagram of literature search. ${ }^{*}$ Two RCTs $[27,28]$ were considered as one study as they used the same population.

(Chapters VII and VIII, $n=1$ ). Among the 36 individual conditions included in these studies, the most frequently researched conditions were breech presentation $(n=7$; $[14,16,17,67-70])$, and tumors $(n=3$; [27-29]).

3.3. Moxibustion Interventions and Control Groups. There are various methods of moxibustion treatment, including direct and indirect treatment with moxa cones, moxa sticks, moxa rolls with herbs, and natural moxibustion. We classified the treatments based on the World Health Organization (WHO) international standard terminologies on traditional medicine [6]. Direct and indirect moxibustion using a moxa cone was the most commonly used method in the studies included in this review. Direct moxibustion, in which an ignited moxa cone is applied directly to the skin surface at the acupuncture point [6], was used in seven studies [26, 39, 42, 48, 59,
61, 66]. In addition, indirect moxibustion, performed by placing some insulating material (ginger, salt, garlic, etc., according to symptoms) between the moxa cone and the skin [6], was used in 19 studies [29, 30, 32, 36, 40, 45, 47, 49$52,54,56,58,60,62-65]$. Moxa stick moxibustion involves the use of ignited moxa sticks named according to the type of stimulation method used (gentle, circling, pecking sparrow, and suspended moxibustion) [6] and was used in 19 studies $[14,16,17,27,28,31,34,35,37,41,44,46,53,55,57,67-$ 70]. Moxa rolls with herbs (Taiyi moxa stick) were used in one study [33]. In addition, natural moxibustion, known as vesiculation moxibustion, in which irritants are applied at the acupuncture points to produce blistering and local congestion [6], was used in one study [43]. One study [38] employed multiple moxibustion techniques. The most frequently used acupuncture point was ST36 $(n=13)$, 


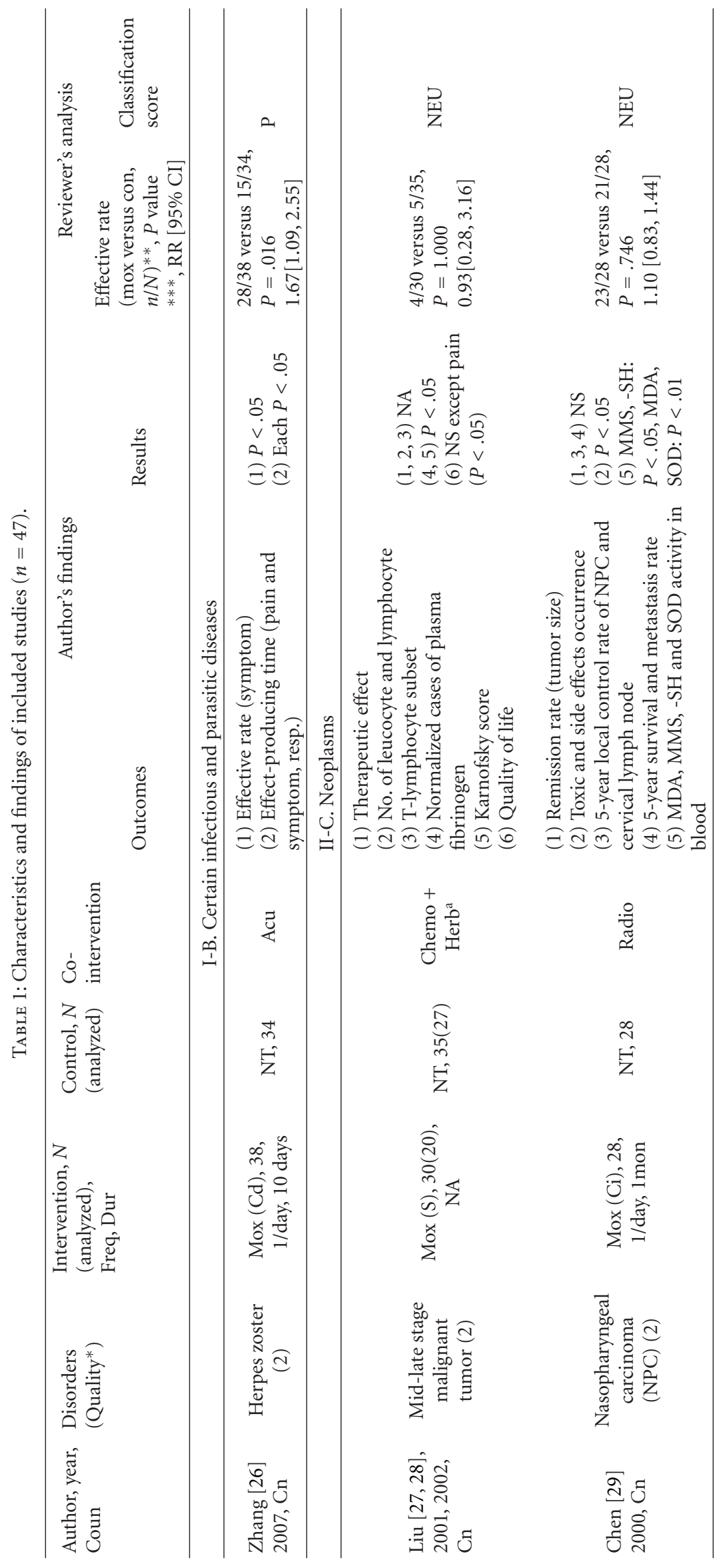




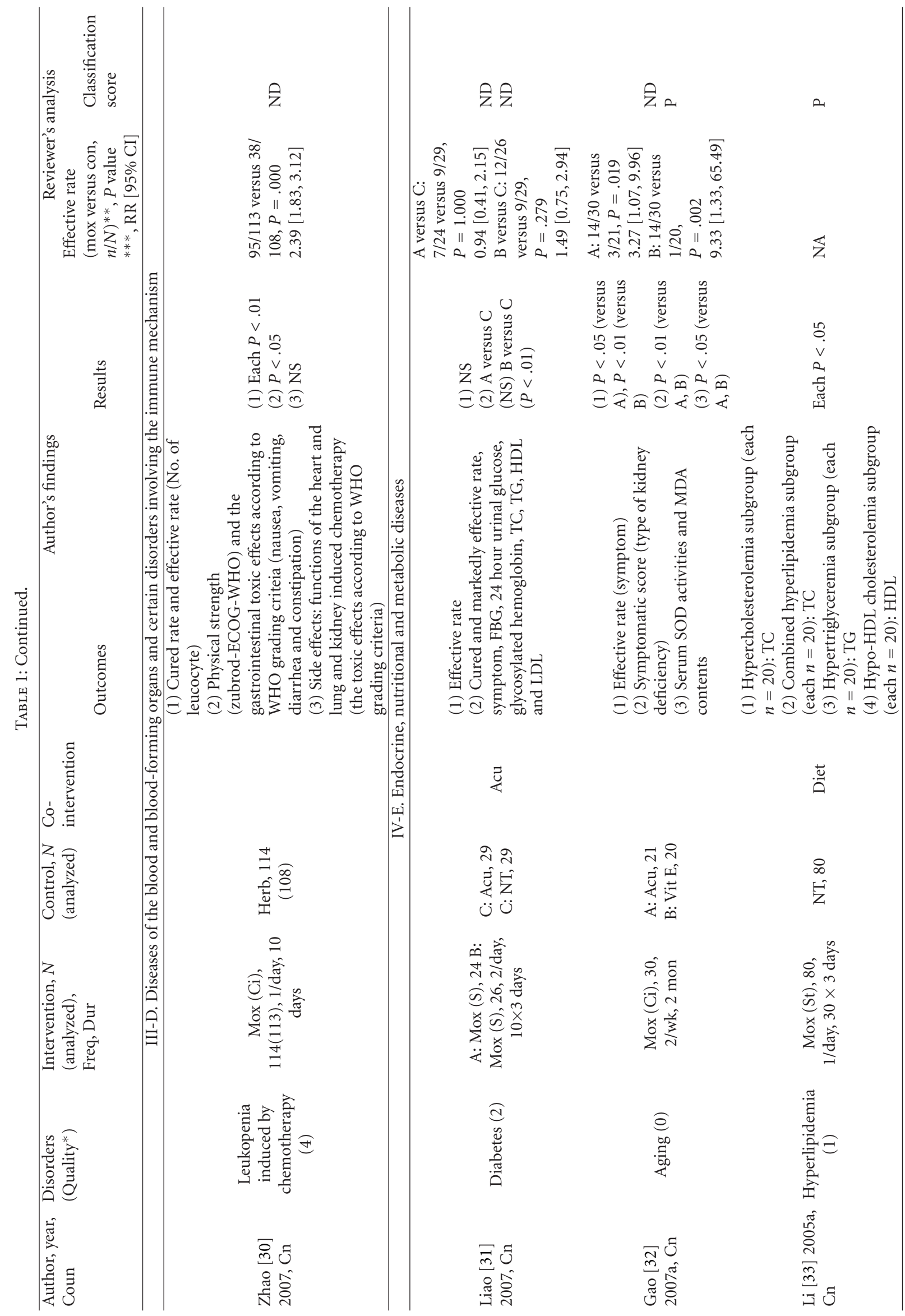




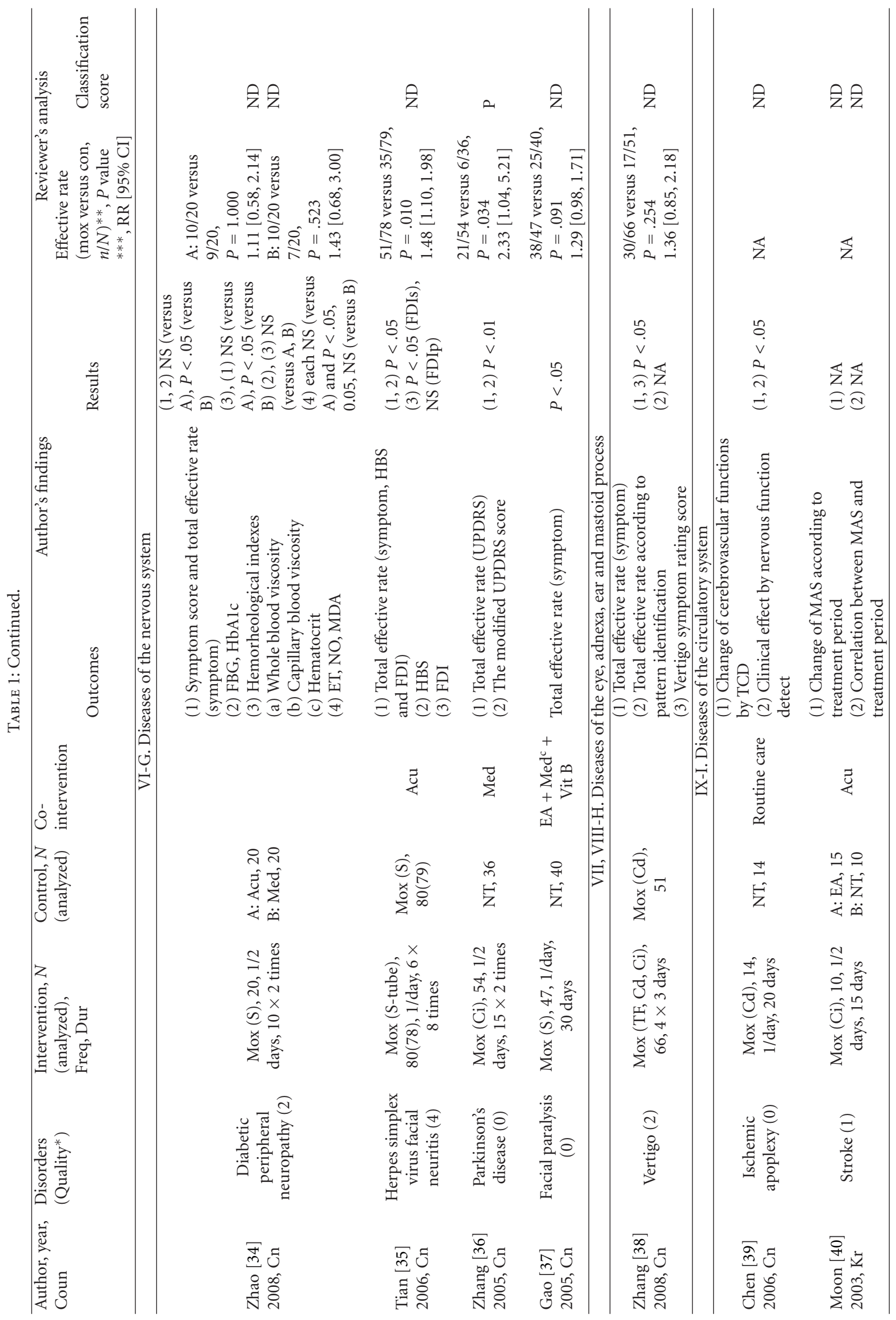




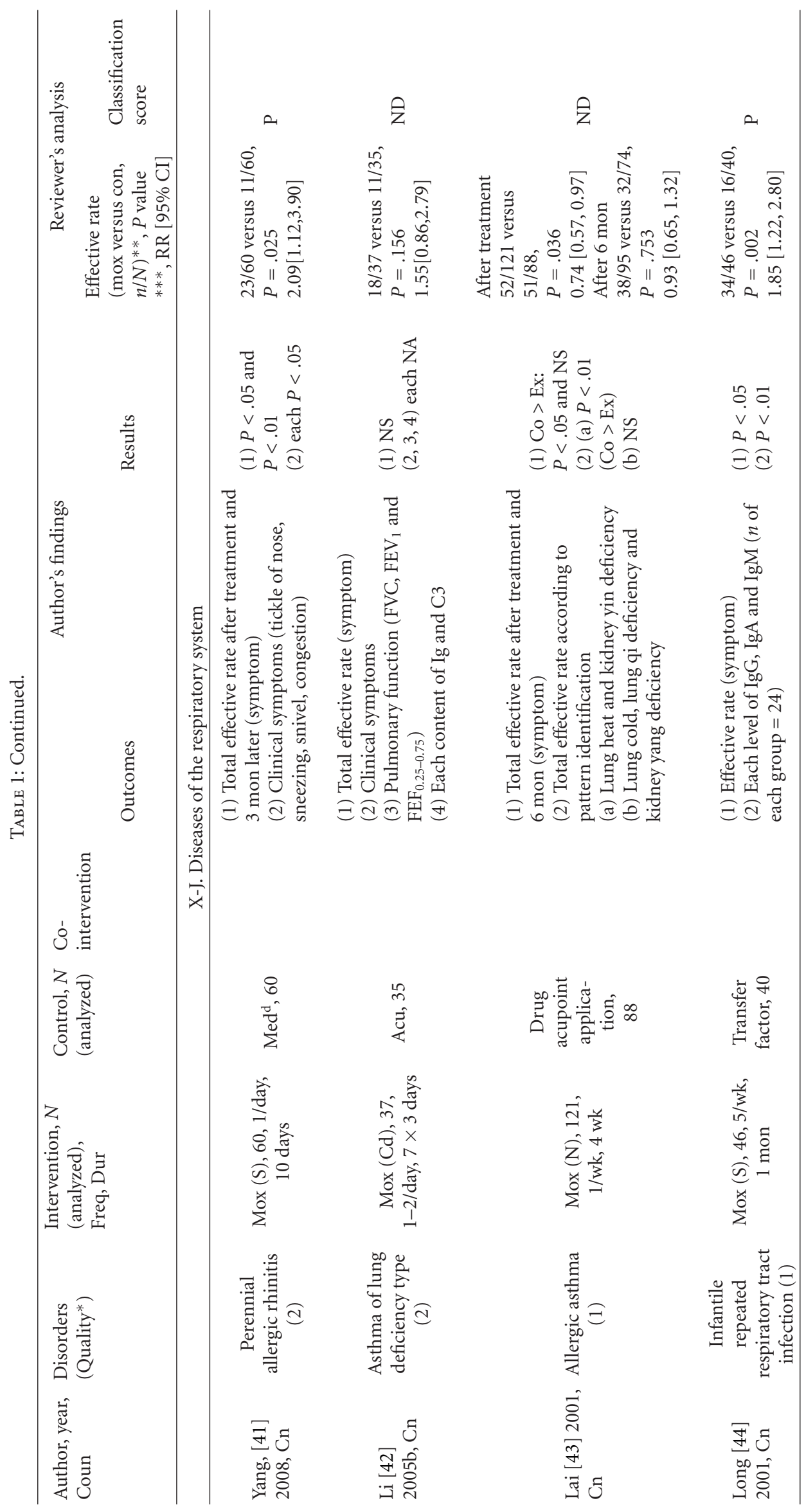




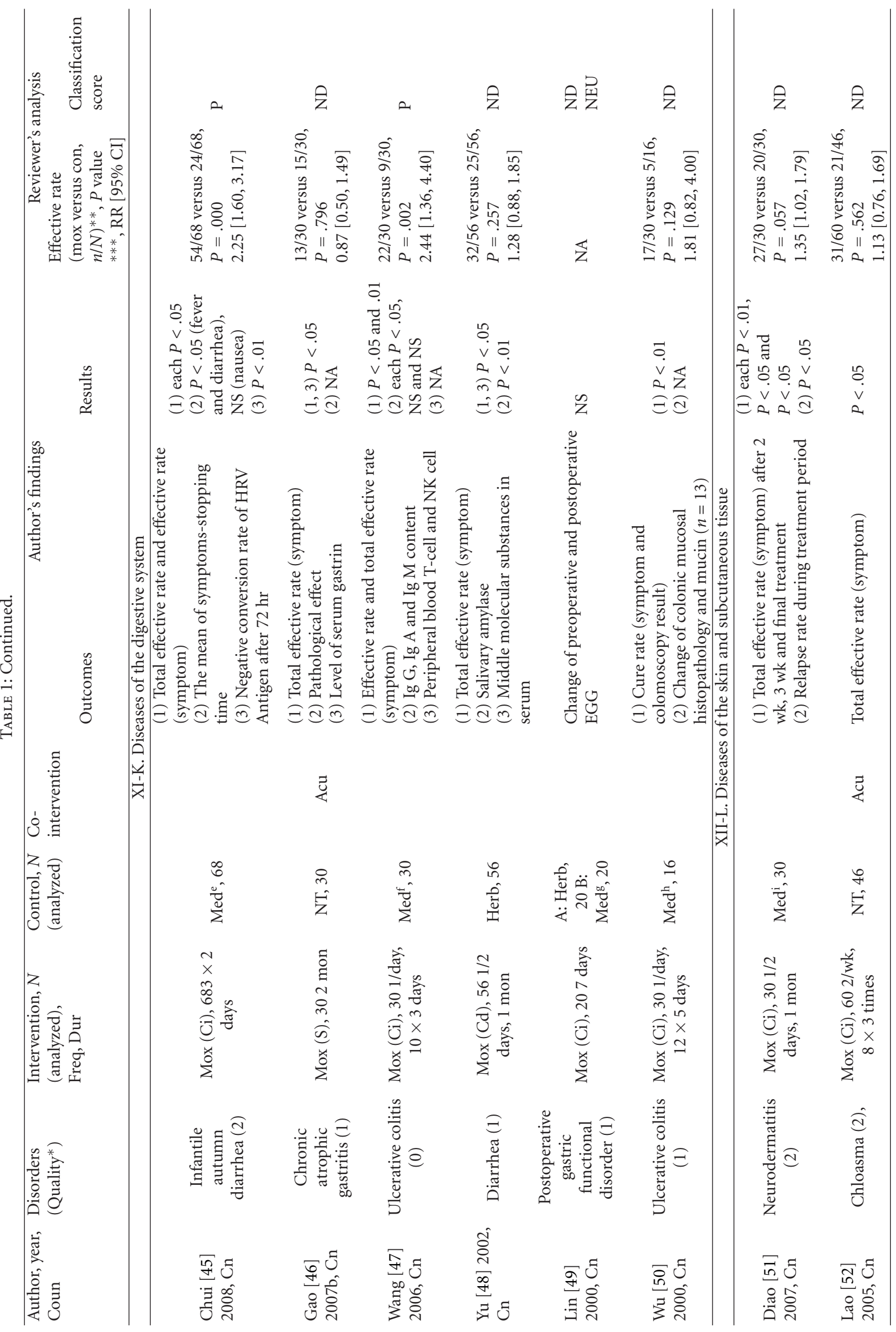




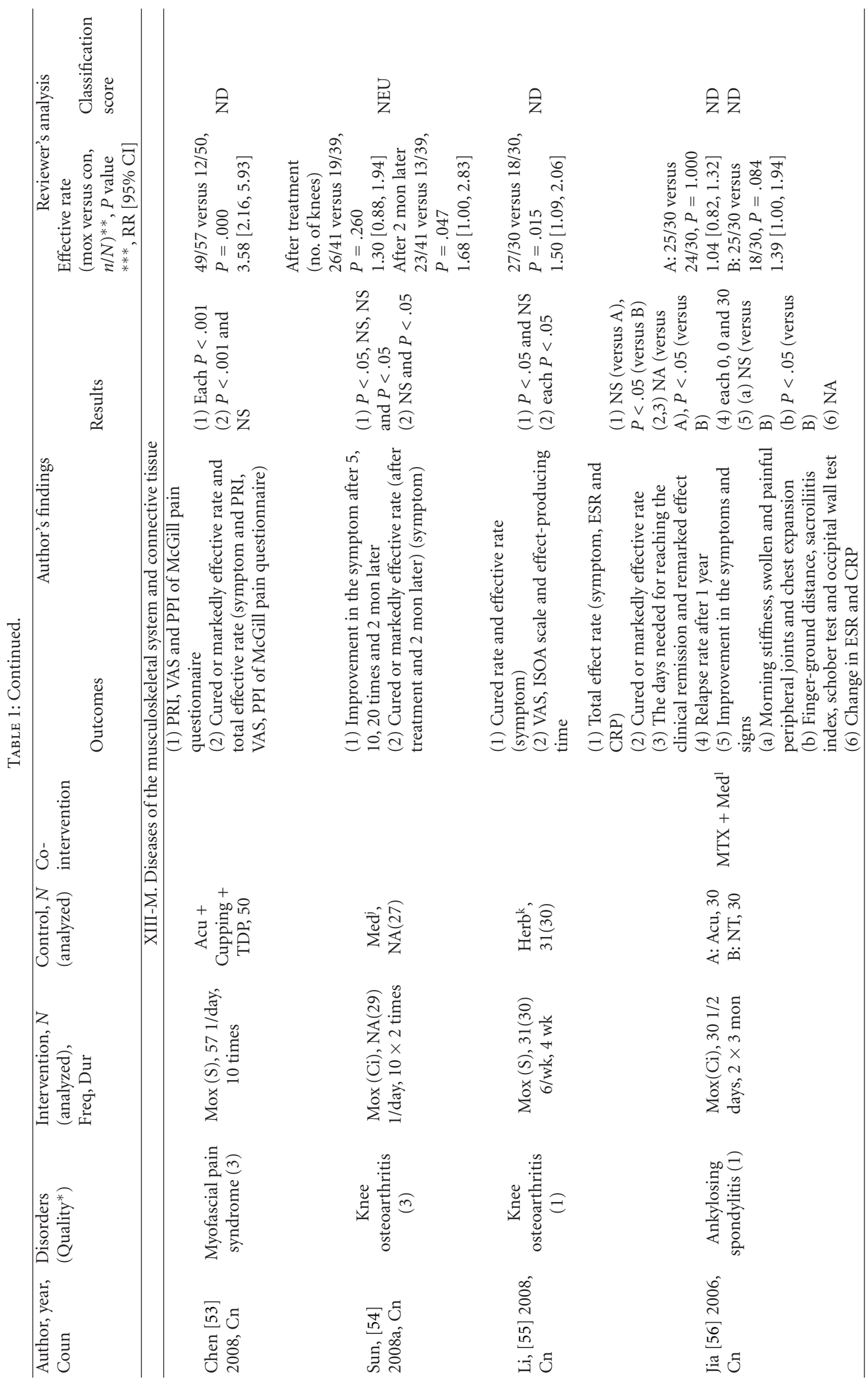




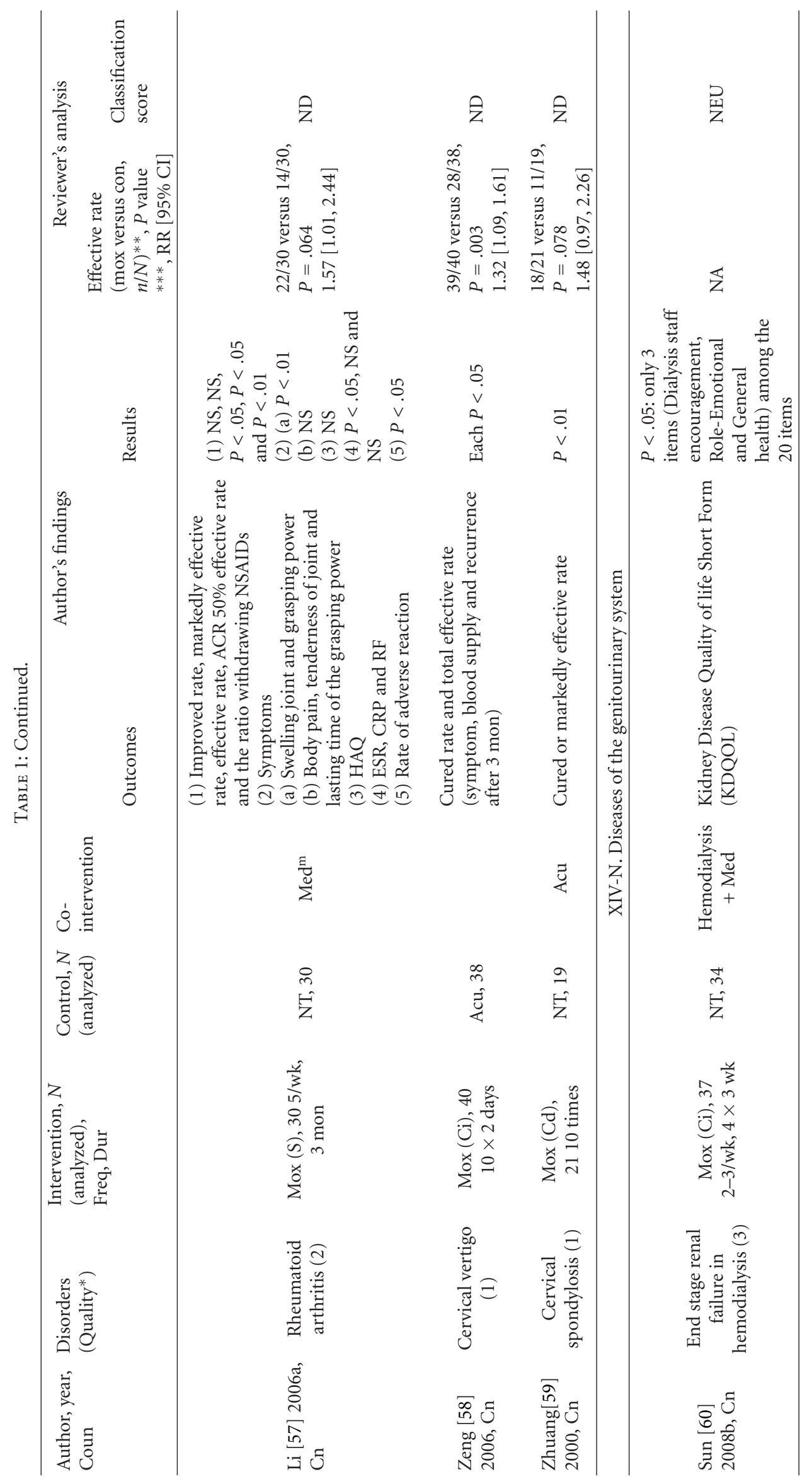




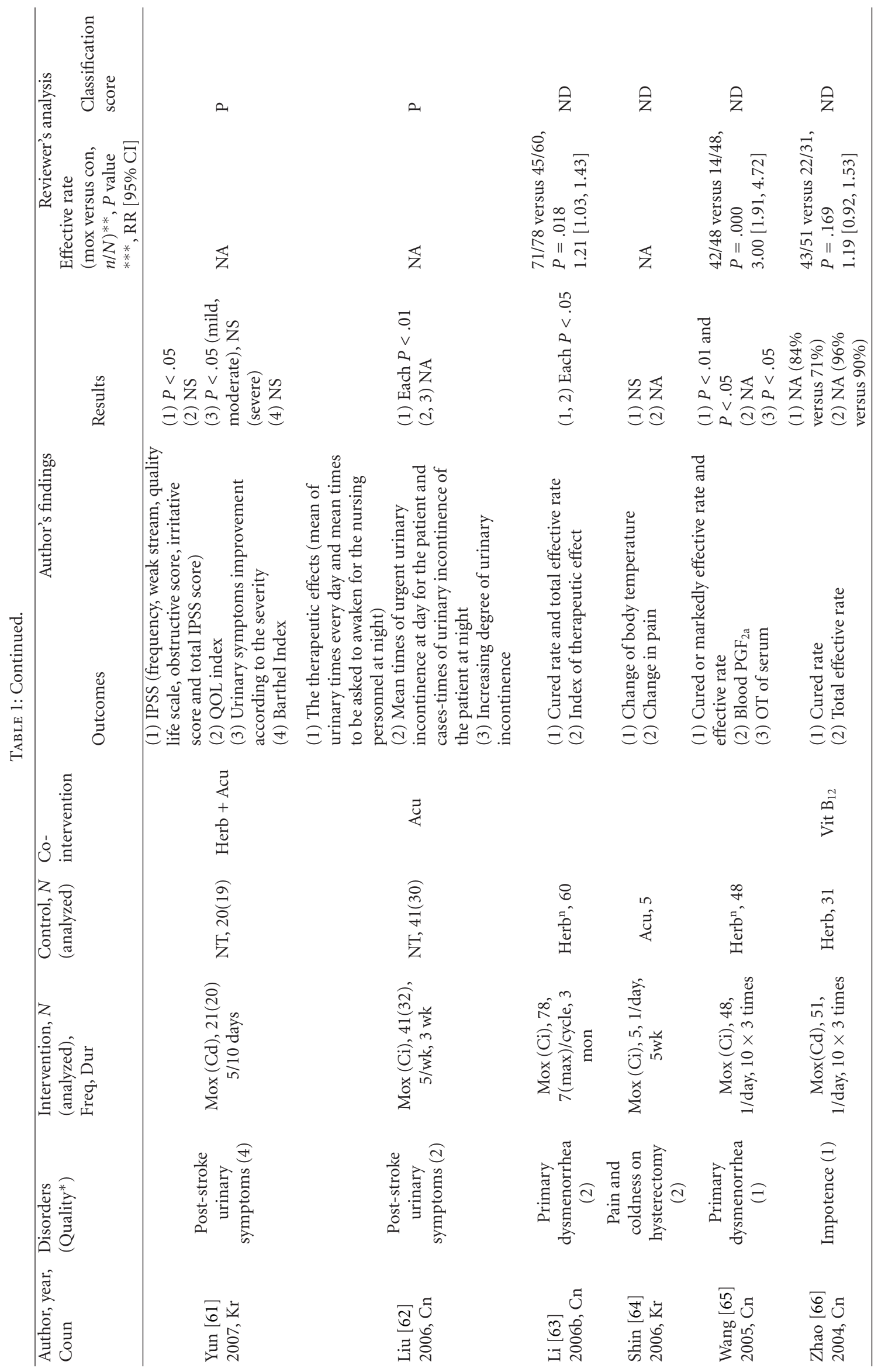




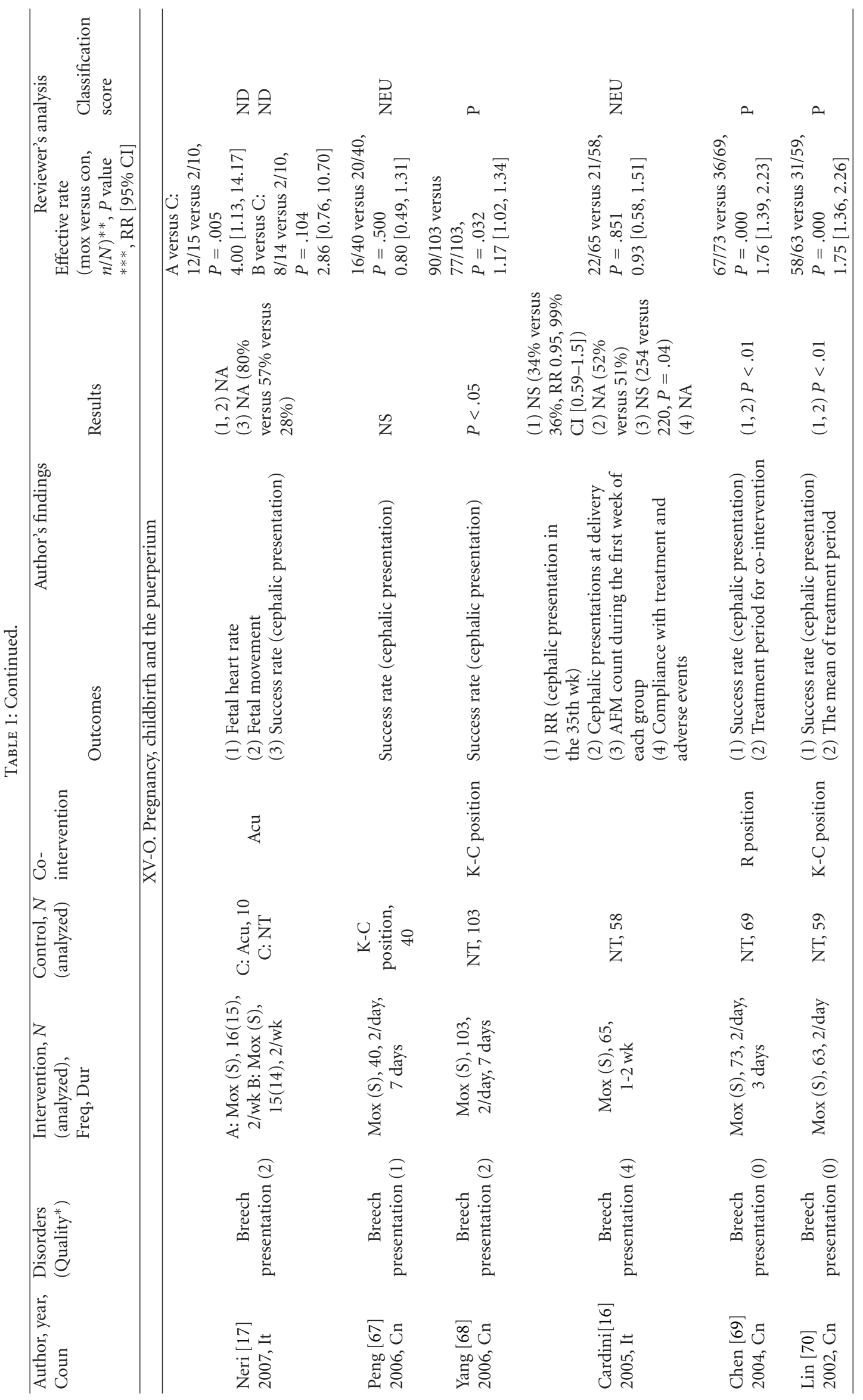




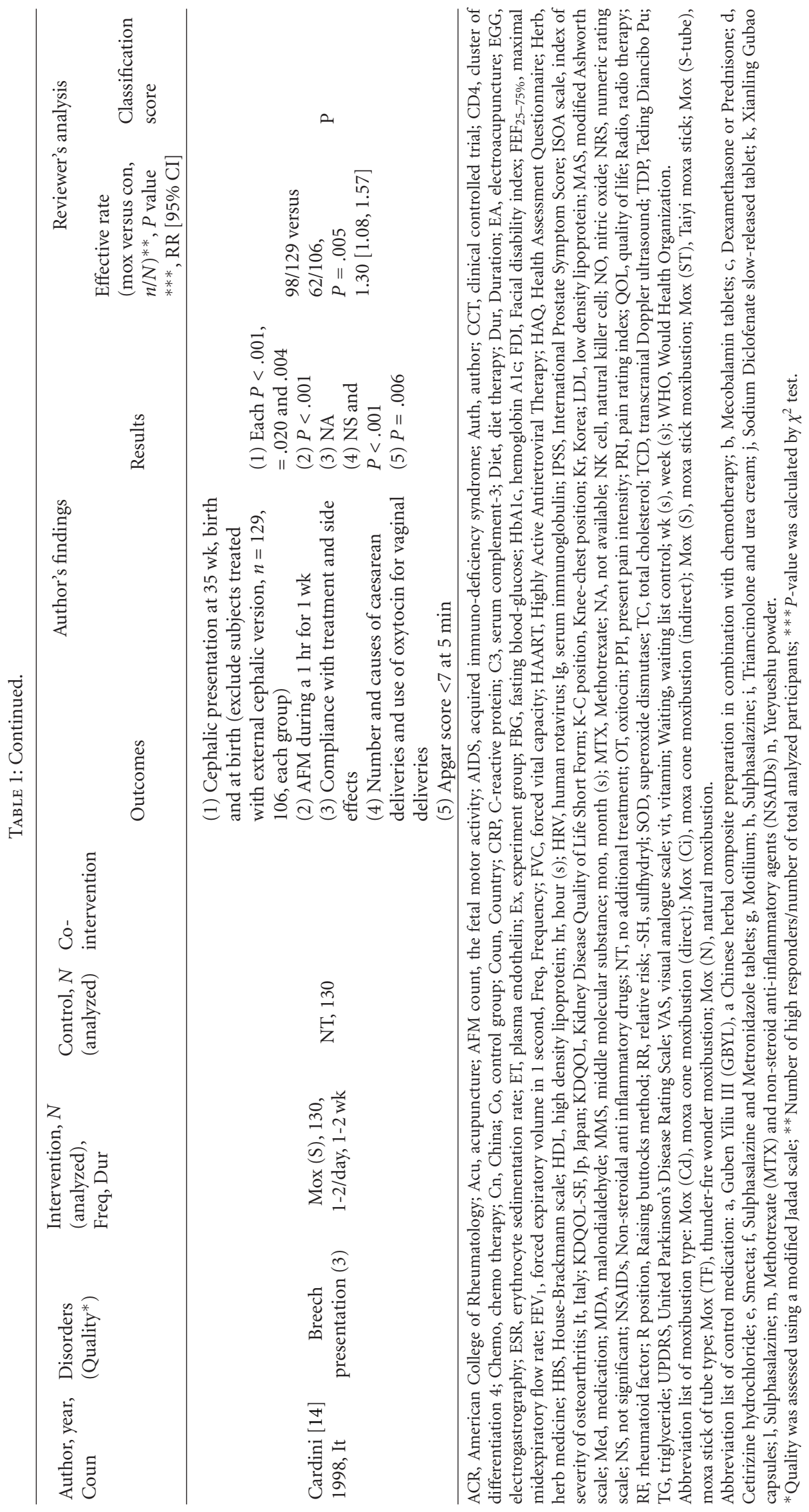


followed by CV8 $(n=11), \operatorname{BL} 23(n=10), \operatorname{BL} 20(n=8)$, CV4 $(n=7), \operatorname{GV} 14(n=7), \operatorname{BL67}(n=7), \operatorname{SP} 6(n=5), \operatorname{BL} 13(n=$ $4)$, and BL18 $(n=4)$. Participants received treatments 4 to 90 times (median, 20).

Three types of control group were included in the studies: (i) general care $(n=11)$, such as Western medication $(n=8)$, vitamin therapy $(n=1)$, injection of transfer factor $(n=1)$, and posture care $(n=1)$; (ii) oriental medical therapy $(n=$ $20)$, such as acupuncture $(n=9)$, herbal medicine $(n=7)$, or combined traditional therapies $(n=4)$; and (iii) no treatment $(n=23)$, such as being placed on a waiting list $(n=2)$ or no additional treatment with the co-intervention $(n=21)$. There were no studies in which treatment effects were compared to a sham-control with indistinguishable appearance and no physiological effect.

3.4. Methodological Quality. Total 47 RCTs were included in this review. The scores for the methodological quality of the RCTs varied from 0 to 4 . Most suffered from poor methodological quality. None of the RCTs were given the maximum of 5 points on the modified Jadad scale. Seven $[16,30,35,53,54,60,61]$ of eight studies with more than 3 points for quality were published after 2005. Seven trials used a single blind method (patient or assessor), and there were no double (patient and assessor) blind studies included in the review. Only 31 RCTs described the method of randomization and nine used an inappropriate method, such as allocation according to treatment order. Power analysis was reported in only one study [16].

3.5. Outcomes. Eighty-three percent of the studies included in this review reported an effective rate (39 of 47 studies), and these were included in the secondary analysis comparing RRs among groups (Table 1). The classification score for the overall effects was decided considering both the authors' and the reviewers' interpretations. According to the classification score, moxibustion was superior to the control in 14 of 54 control group in 47 studies (26\%). There were no statistically significant differences between groups in 7 studies (13\%). The outcome direction was not determined in 33 studies $(61 \%)$ for the following four reasons: (i) inappropriate outcome measures for the indicated disorder; (ii) details of the outcome measures were not described; (iii) the results were too complex to be determined; or (iv) inappropriate control to estimate the effectiveness. Among the 8 studies classified as high quality on the Jadad scale $[14,16,30,35$, $53,54,60,61]$, three studies could not be determined the direction of outcome, due to inappropriate control. Only five studies were estimated their classification score as two studies were positive, and three neutral. In details of high quality studies, indirect moxibustion for 20 days did not improve the symptoms of osteoarthritis compared to medication after treatment, but not in 2 months later, follow up point [54]. Patients for end stage renal failure in hemodialysis were measured by Kidney Disease Quality of life, but moxibustion didn't have additional effect with medication [60]. One study [61] for post-stroke urinary symptoms showed additional effect with oriental medical therapy as co-intervention, and this result was not contradictory to other low quality study
[62]. Other two high qulity studies $[14,16]$ for breech presentation were attempted to polling, but not suitable as heterogeneity.

In detail, 10 days of direct moxibustion generated additional therapeutic effects to acupuncture in cases of herpes zoster [26] (RR (95\% CIs), 1.67 (1.09-2.55), $P=$ .016). Moxibustion showed no additional effects when used in conjunction with chemotherapy or radiotherapy in midto late-stage malignant tumors $[27,28]$ or nasopharyngeal carcinoma [29]. Biweekly indirect moxibustion for 2 months had an anti-aging effect compared to vitamin E (9.33 (1.33, 65.49), $P=.002$ ) [32]. For hyperlipidemia [33], Taiyi moxibustion for 3 months improved the patient response to diet therapy in terms of cholesterol, triglyceride, and highdensity lipoprotein (HDL) levels. In Parkinson's disease, 30 courses of indirect moxibustion improved the effectiveness of medication based on the Unified Parkinson's Disease Rating Scale score (2.33 (1.04-5.21), $P=.034)$ [36]. Stick moxibustion for 1 month did not improve the effects of a mixed therapy (electroacupuncture, medication, and vitamins) in facial paralysis [37], and 20 courses of stick moxibustion were not superior to medication in the treatment of diabetic peripheral neuropathy [34]. Direct moxibustion for 20 days provided additional improvement in the treatment of ischemic apoplexy, as determined by clinical symptoms and changes in transcranial Doppler (TCD) findings [39]. For allergic rhinitis and infantile repeated respiratory tract infection, moxa stick application for 10 days or 1 month was more effective than medication (2.09 (1.12-3.90), $P$ $=.025)[41]$ or intramuscular injection of transfer factor (1.85 (1.22-2.80), $P=.002$ ) [44]. Moxibustion for 6 days was superior to medication in infantile autumn diarrhea (2.25 (1.60-3.17), $P<.001)$ [45]. In patients with chronic atrophic gastritis, moxibustion did not show an additional effect to acupuncture [46]. For ankylosing spondylitis [56], rheumatoid arthritis [57] and cervical spondylosis [59], moxibustion did not show additional benefits.

Only seven studies were performed for statistical pooling (Figure 2). Indirect moxibustion for 1 or 3 months was more effective than medication in two ulcerative colitis studies (2.20 (1.37, 3.52), $P=.001, I^{2}=0 \%, P$ for heterogeneity $=.55)$ (Figure $2(\mathrm{a}) ;[47,50])$. Moxa stick treatment for $1-2$ weeks did not show a effect on breech presentation compared to no treatment control $\left(1.19(0.88,1.60), P=.26, I^{2}=40 \%\right.$, $P$ for heterogeneity $=.20$ ) in two studies (Figure $2(\mathrm{~b}) ; 14,16)$. Moxa stick had additional effect to the posture care $(1.51$ (1.10, 2.08), $P=.01)$, although marked heterogeneity was observed in this model $\left(I^{2}=86 \%, P\right.$ for heterogeneity = .0007 , Figure 2(c); [68-70]).

The direction of study outcome (positive, neutral, not determined, or negative) was not significantly associated with intervention type (moxa cone versus moxa stick versus others, $\chi^{2}=1.835$, $\mathrm{df}=4, P=.895$ ), country of origin (East Asia versus others, $\left.\chi^{2}=.572, \mathrm{df}=2, P=.758\right)$, or language (English versus other language, $\chi^{2}=2.573$, $\mathrm{df}=2, P=.392$ ). In case of the association between direction of study outcome and study quality (high versus low, as assessed using the modified Jadad scale $)$ seems to be near to $P<.05\left(\chi^{2}=5.222\right.$, $\mathrm{df}=2, P=.084)$, This may be caused by biased results that 


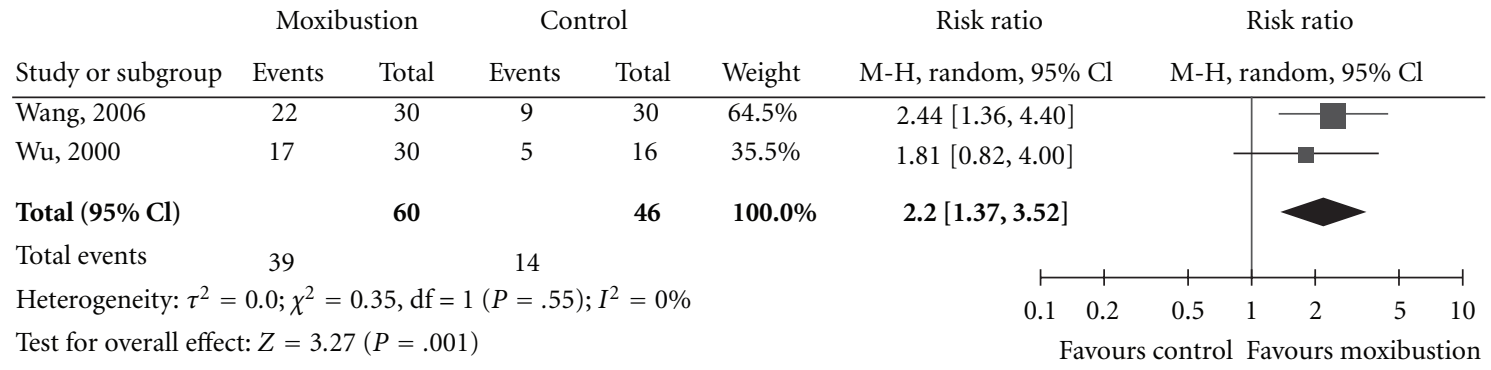

(a)

Treatment Control

Risk ratio

Risk ratio

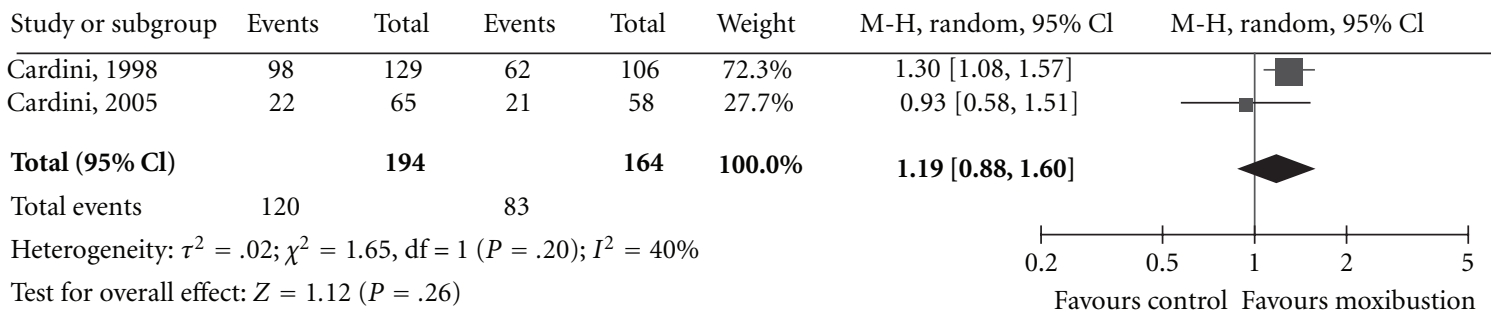

(b)

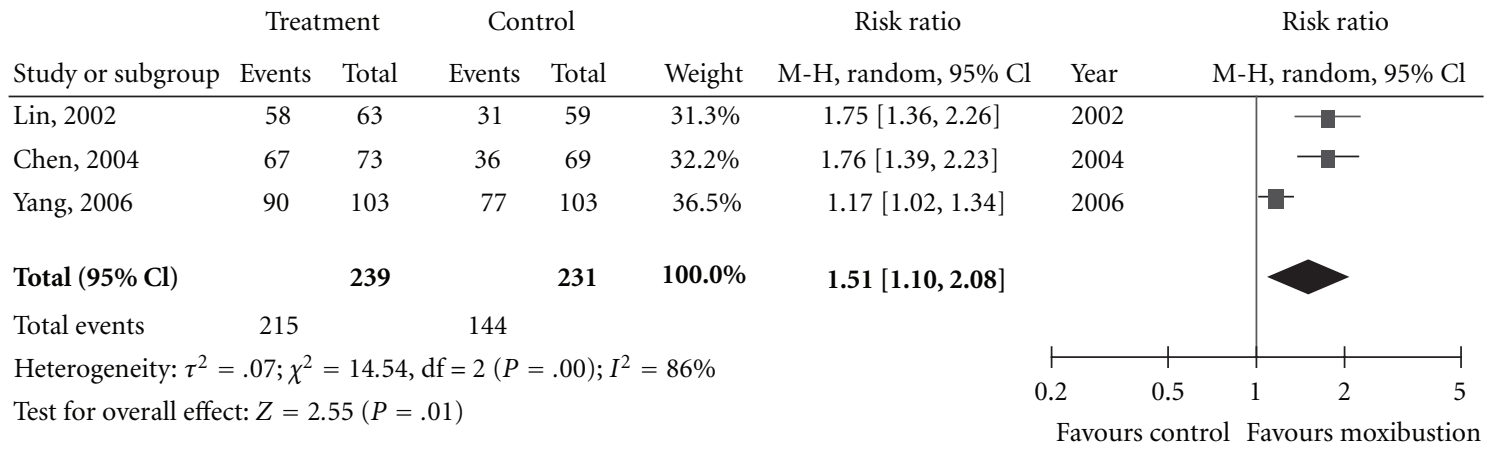

(c)

FIGURE 2: Forest plot of moxibustion compared to control. (a) Moxibustion compared with medication in ulcerative colitis [46, 49]. (b) Moxibustion compared with waiting-list control in breech presentation [14,16]. (c) Moxibustion plus postual care compared with postual care only in breech presentation [68-70].

studies of low quality show a tendency of ND or positive. There was significant association between the direction of study outcome and control type (general care versus oriental medical therapy control versus no additional treatment with or without co-intervention, $\chi^{2}=21.209, \mathrm{df}=4, P<$ $.001)$. It might be caused by inappropriate control, such as oriental medical therapy. Moxibustion studies compared to oriental medical therapy were assessed as ND. When sensitivity analysis were performed excluding "ND", the direction of study outcome (positive, neutral, or negative) was not significantly associated with control group (general care versus no additional treatment with or without cointervention, $\left.\chi^{2}=.936, \mathrm{df}=2, P=.713\right)$.

3.6. Adverse Effects. Only 12 studies $[14,16,29,30,51,52$, $55,57,61,62,67,68]$ commented on adverse events. Seven studies $[14,51,52,55,61,67,68]$ reported no adverse event associated with moxibustion during the treatment period.
Patients participating in a rheumatoid arthritis study [57] showed a slight and reversible increase in aminotransferase levels (3 of 30 cases) and a slight reduction in leukocyte count (two cases) after treatment with moxa sticks, whereas those in the control group (treatment with methotrexate and NSAIDs) had no appetite (nine cases), abnormal taste (nine cases), nausea (eight cases), increased aminotransferase levels (six cases) and thrombocytopenia (five cases). Liu et al. [62] reported slight burning and blistering in two patients treated with indirect moxibustion for poststroke urinary tract symptoms. The two studies in which moxibustion was applied to nasopharyngeal carcinoma [29] and chemotherapy-induced leukopenia [30] also reported toxic side effects, but these were induced by radiotherapy or chemotherapy, rather than moxibustion. In the study on breech presentation, Cardini and weixin [14] reported no adverse event associated with moxibustion, and the numbers of cases of premature rupture of the membranes (PROM) 
and preterm delivery were less in the moxibustion group compared to the waiting list control (4 cases versus 12 cases of PROM and two cases versus three cases of preterm delivery among 130 subjects, resp.) [14]. However, in another study performed by the same group [16], two cases of preterm delivery possibly due to PROM and one case of bleeding were noted in association with moxibustion, whereas one case of preterm delivery occurred in the waiting list control group. In this study, moxibustion was associated with complaints of unpleasantness and physical disturbances due to the odor (42\%), throat problems (22\%), and abdominal pain because of contractions (17\%). Twenty-two percent of participants temporarily or definitively interrupted the treatment because of these symptoms. Peng's study [67] for breech presentation reported five cases of abdominal pain in control group.

\section{Discussion}

Although many studies have provided encouraging results regarding the use of moxibustion in various disorders, definitive conclusions cannot be drawn from the evidence presented in this review. The use of moxibustion has been studied in a wide range of diseases, from neoplasm to pain, but we cannot properly evaluate the effectiveness of moxibustion in each disease due to the limited number of corresponding studies. In addition, the overall quality of these studies was low, and the use of controls was inappropriate. Nevertheless, meta-analysis suggested beneficial effect that moxibustion appeared to be more effective than medication in ulcerative colitis. In breech presentation, there is strong heterogeneity exist, thus the results are inconclusive. There have been three relevant systematic reviews on this topic, however, the results are not directly comparable because two were not focused on the moxibustion as an intervention $[21,22]$, and one missed the relevant studies included in our review [20].

Among the 36 individual conditions included in these studies, the most frequently studied were breech presentation ( $n=7 ;[14,16,17,67-70])$, and tumors $(n=3 ; 27-29)$. As only one or two studies covered each disease, definitive conclusions cannot be drawn.

We found that the quality of studies regarding moxibustion was unsatisfactory. Thirty-nine of 47 studies (83\%) received scores of less than 3 on a modified Jadad scale. In most studies, the details of the moxibustion procedure used were not fully described. Methods regarding randomization were unclear and power analysis was seldom reported. Concealment of allocation and blinding methods were not clearly described, and details regarding drop-out and withdrawal rates were often insufficient. Even the trials that scored as high quality on the modified Jadad scale were not devoid of flaws; for example, none of the studies attempted patient blinding and only one study reported power analysis; several studies that had been expressed as positive in their original papers were re-classified as ND because the outcome measures were not appropriate either for the disease or the results were contradictory; seventy-four percent of studies did not report adverse events related to intervention. Despite these shortcomings, we found that methodological quality has improved recently, and seven of the eight high-quality studies were published after 2005.

All of the studies used inappropriate controls, which makes evaluation difficult because controls in RCTs provide important insight into the effectiveness of the treatment and help to eliminate factors that may otherwise skew the results [71]. Furthermore, it is extremely difficult to compare each other and estimate their effectiveness because of the inappropriateness in the quality and control. In our analysis, 20 studies, which had been compared to oriental medical therapy, were re-classified as ND because the controls were not appropriate to estimate effectiveness. The issue of appropriate control methods for moxibustion has not drawn a great deal of attention. Since many components, such as heat, fumes, and moxa extract [8], may contribute to the therapeutic effect, developing appropriate controls for moxibustion is not easy tasks. Two types of sham moxibustion designed to isolate heat $[72,73]$ have been introduced. However, it is not obvious whether subjects were really blinded because the thermal intensity was different. Therefore, there is a need for a more appropriate sham moxibustion method for use as a control.

Among the 12 RCTs that reported adverse events, none reported serious adverse events. There were complaints related to unpleasantness and physical disturbances due to the odor [16]. Blistering and slight burning were also reported in one studies [62]. Because moxibustion involves thermal stimulation of the skin over a prolonged period, it is important that only experienced and well-trained practitioners provide this therapy [62]. In addition, for widespread adoption of moxibustion, it will be necessary to develop an odor-free device for its application.

This review had several limitations. Although we made efforts to retrieve all relevant RCTs, some studies in other databases may have been missed. However, it is reported that Pubmed offers better indexing and more search features, and there is overall a relatively high degree of overlap between Medline and EMBASE and CENTRAL [23, 74]. Further limitations include the paucity and the overall insufficient methodological quality of the primary data. As the quality of clinical trials and reporting methodologies included in this review was generally weak, further high-quality trials are needed to assess the effectiveness of moxibustion in treating several diseases. In this regard, future trials should adhere to rigorous trial designs that are suitable for the research questions. To improve the research quality, future researchers should follow the guidelines for reporting clinical trials, such as the Consolidated Standards of Reporting Trials (CONSORT) statement. We suggest that specific guidelines for the reporting of moxibustion trials, similar to the Reporting Interventions in Controlled Trials of Acupuncture (STRICTA) [75], should be developed and followed by researchers.

In conclusion, even though our results for the effectiveness of moxibustion are not conclusive due to the heterogeneity and the poor quality of the studies, we intend that this review gives researchers and clinicians more information of the real clinical usages of moxibustion, and thus it could be extended over a wide field of disorder for practice and 
research. Of course, more specific and rigorous trials with large sample sizes are needed to evaluate the effectiveness of moxibustion for each disease.

\section{Funding}

This research was supported by Basic Science Research Program through the National Research Foundation (NRF) funded by the Ministry of Education, Science and Technology (R11-2005-014).

\section{Acknowledgment}

The authors thank Mr Soo Min Choi and Sungjin Lee for the proof reading of the table and revision, respectively.

\section{References}

[1] J. J. Y. Sung, "Acupuncture for gastrointestinal disorders: myth or magic," Gut, vol. 51, no. 5, pp. 617-619, 2002.

[2] G. Bodeker and F. Kronenberg, "A public health agenda for traditional, complementary, and alternative medicine," American Journal of Public Health, vol. 92, no. 10, pp. 15821591, 2002.

[3] T. Hesketh and W. X. Zhu, "Health in China: traditional Chinese medicine: one country, two systems," British Medical Journal, vol. 315, no. 7100, pp. 115-117, 1997.

[4] C. H. Han, M. S. Shin, S. H. Shin, K. W. Kang, S. H. Park, and S. M. Choi, "Telephone survey for grasping clinical actual state of moxibustion therapeutics in Korea," Journal of Meridian and Acupoint, vol. 24, pp. 17-31, 2007 (Korean).

[5] C. H. Han, M. S. Shin, S. H. Shin, K. W. Kang, B. K. Kang, S. H. Park et al., "An in-depth interview for use of moxibustion therapy in Korea," Journal of Meridian and Acupoint, vol. 25, pp. 85-97, 2008 (Korean).

[6] World Health Organization Western Pacific Region, WHO International Standard Terminologies on Traditional Medicine in the Western Pacific Region, WHO Regional Office for the Western Pacific, Manila, Philippines, 2007.

[7] S. Joos, B. Brinkhaus, C. Maluche et al., "Acupuncture and moxibustion in the treatment of active Crohn's disease: a randomized controlled study," Digestion, vol. 69, no. 3, pp. 131-139, 2004.

[8] H. Yamashita, Y. Ichiman, and Y. Tanno, "Changes in peripheral lymphocyte subpopulations after direct moxibustion," The American Journal of Chinese Medicine, vol. 29, pp. 227$235,2001$.

[9] S. T. Im, K. H. Kim, and K. S. Kim, "A study of physical characteristics of moxibustion," The Journal of Korean Acupuncture and Moxibustion Society, vol. 11, pp. 327-336, 1994 (Korean).

[10] S. B. Kang, Medical Classics of Acupuncture and Moxibustion, Iljoongsa, Seoul, Republic of Korea, 2000.

[11] Y. T. Choi, Acupuncture and Moxibustion, Jip-Moon-Dang, Seoul, Republic of Korea, 2001.

[12] Y.-Y. Kung, F.-P. Chen, and S.-J. Hwang, "The different immunomodulation of indirect moxibustion on normal subjects and patients with systemic lupus erythematosus," American Journal of Chinese Medicine, vol. 34, no. 1, pp. 47$56,2006$.

[13] X. Shen, G. Ding, J. Wei et al., "An infrared radiation study of the biophysical characteristics of traditional moxibustion," Complementary Therapies in Medicine, vol. 14, no. 3, pp. 213219, 2006.
[14] F. Cardini and H. Weixin, "Moxibustion for correction of breech presentation: a randomized controlled trial," Journal of the American Medical Association, vol. 280, no. 18, pp. 15801584, 1998.

[15] Y. Kanakura, K. Kometani, T. Nagata, K. Niwa, H. Kamatsuki, Y. Shinzato et al., "Moxibustion treatment of breech presentation," The American Journal of Chinese Medicine, vol. 29, pp. 37-45, 2001.

[16] F. Cardini, P. Lombardo, A. L. Regalia et al., "A randomised controlled trial of moxibustion for breech presentation," British Journal of Obstetrics and Gynaecology, vol. 112, no. 6, pp. 743-747, 2005.

[17] I. Neri, V. De Pace, P. Venturini, and F. Facchinetti, "Effects of three different stimulations (acupuncture, moxibustion, acupuncture plus moxibustion) of BL.67 acupoint at small toe on fetal behavior of breech presentation," The American Journal of Chinese Medicine, vol. 35, pp. 27-33, 2007.

[18] R. Sakakibara, E. Murakami, A. Katagiri, S. Hayakawa, T. Uchiyama, T. Yamamoto et al., "Moxibustion, an alternative therapy, ameliorated disturbed circadian rhythm of plasma arginine vasopressin and urine output in multiple system atrophy," Annals of Internal Medicine, vol. 46, pp. 1015-1018, 2007.

[19] M. Mitchell and K. Allen, “An exploratory study of women's experiences and key stakeholders views of moxibustion for cephalic version in breech presentation," Complementary Therapies in Clinical Practice, vol. 14, pp. 264-272, 2008.

[20] M. E. Coyle, C. A. Smith, and B. Peat, "Cephalic version by moxibustion for breech presentation," Cochrane Database of Systematic Reviews, no. 2, Article ID CD003928, 2005.

[21] I. van den Berg, J. L. Bosch, B. Jacobs, I. Bouman, J. J. Duvekot, and M. G. M. Hunink, "Effectiveness of acupuncture-type interventions versus expectant management to correct breech presentation: a systematic review," Complementary Therapies in Medicine, vol. 16, no. 2, pp. 92-100, 2008.

[22] X. Li, J. Hu, X. Wang, H. Zhang, and J. Liu, "Moxibustion and other acupuncture point stimulation methods to treat breech presentation: a systematic review of clinical trials," Chinese Medicine, vol. 4, article no. 4, pp. 1-8, 2009.

[23] E. Cogo, M. Sampson, I. Ajiferuke et al., "Searching for controlled trials of complementary and alternative medicine: a comparison of 15 databases," Evidence-Based Complementary and Alternative Medicine. In press.

[24] A. R. Jadad, R. A. Moore, D. Carroll et al., "Assessing the quality of reports of randomized clinical trials: is blinding necessary?" Controlled Clinical Trials, vol. 17, no. 1, pp. 1-12, 1996.

[25] A. R. White and E. Ernst, "A systematic review of randomized controlled trials of acupuncture for neck pain," Rheumatology, vol. 38, no. 2, pp. 143-147, 1999.

[26] M. Zhang, L. Qiu, and J. Zhang, "Observation on therapeutic effect of surround needling plus surround moxibustion on herpes zoster," Zhongguo Zhen Jiu, vol. 27, no. 2, pp. 123-125, 2007 (Chinese).

[27] J. Liu, R. C. Yu, and W. J. Tang, "Influence of combined therapy of guben yiliu III, moxibustion and chemotherapy on immune function and blood coagulation mechanism in patients with mid-late stage malignant tumor," Zhongguo Zhong Xi Yi Jie He Za Zhi, vol. 22, pp. 104-106, 2002 (Chinese).

[28] J. Liu, R. C. Yu, and X. Q. Rao, "Study on effect of moxibustion and guben yiliu III combined with chemotherapy in treating middle-late stage malignant tumor," Zhongguo Zhong Xi Yi Jie He Za Zhi, vol. 21, no. 4, pp. 262-264, 2001 (Chinese). 
[29] K. Chen, Y. Jiang, and H. Wen, "Clinical study on treatment of nasopharyngeal carcinoma by radio- and chemotherapy with supplementary moxibustion on Shenque point," Zhongguo Zhong Xi Yi Jie He Za Zhi, vol. 20, no. 10, pp. 733-735, 2000 (Chinese).

[30] X. X. Zhao, M. Lu, X. Zhu, P. Gao, Y. L. Li, X. M. Wang et al., "Multi-central clinical evaluation of gingerpartitioned moxibustion for treatment of leukopenia induced by chemotherapy," Zhongguo Zhen Jiu, vol. 27, pp. 715-720, 2007 (Chinese).

[31] H. Liao, P. Xi, Q. Chen, L. Yi, and Y. Zhao, "Clinical study on acupuncture, moxibustion, acupuncture plus moxibustion at Weiwanxiashu (EX-B3) for treatment of diabetes," Zhongguo Zhen Jiu, vol. 27, no. 7, pp. 482-484, 2007 (Chinese).

[32] S. Z. Gao and J. Wang, "Clinical observation on drugseparated moxibustion at the navel for anti-aging," Zhongguo Zhen Jiu, vol. 27, no. 6, pp. 398-402, 2007 (Chinese).

[33] J. P. Li, Y. N. Yao, P. D. He, X. M. Gan, and F. L. Fan, "Clinical study on moxibustion for treatment of abnormal blood lipids," Zhongguo Zhen Jiu, vol. 25, pp. 825-827, 2005 (Chinese).

[34] J. L. Zhao and Z. R. Li, "Clinical observation on mild-warm moxibustion for treatment of diabetic peripheral neuropathy," Zhongguo Zhen Jiu, vol. 28, no. 1, pp. 13-16, 2008 (Chinese).

[35] F. W. Tian, Z. X. Wang, Y. Lu, C. Y. Gou, and H. Wang, "Controlled observation on Guan-moxibustion and suspended moxibustion for treatment of herpes simplex virus facial neuritis," Zhongguo Zhen Jiu, vol. 26, pp. 166-168, 2006 (Chinese).

[36] J. F. Zhang, G. S. Sun, and G. H. Zhao, "Observation on therapeutic effect of herbs-partitioned moxibustion on Parkinson disease of 54 cases," Zhongguo Zhen Jiu, vol. 25, pp. 610-612, 2005 (Chinese).

[37] H. Y. Gao, X. L. Zhang, Y. Wang, and L. F. Yan, "Clinical observation on double moxibustion at Yifeng (TE 17) combined with electroacupuncture for treatment of facial paralysis," Zhongguo Zhen Jiu, vol. 25, pp. 713-714, 2005 (Chinese).

[38] G. A. Zhang, J. Luo, and L. H. Huang, "Observation on clinical therapeutic effect of improved thunder-fire miraculous needle on vertigo," Zhongguo Zhen Jiu, vol. 28, no. 4, pp. 280-282, 2008 (Chinese).

[39] W. Chen, M. Tan, L. A. Liang, and T. L. Weng, "Effects of moxibustion at Zusanli (ST 36) and Xuanzhong (GB 39) on cerebrovascular function in the patient of ischemic apoplexy," Zhongguo Zhen Jiu, vol. 26, pp. 161-165, 2006 (Chinese).

[40] S.-K. Moon, Y.-K. Whang, S.-U. Park et al., "Antispastic effect of electroacupuncture and moxibustion in stroke patients," American Journal of Chinese Medicine, vol. 31, no. 3, pp. 467474, 2003.

[41] S. R. Yang, H. Chen, and Q. Xie, "Observation on therapeutic effect of moxibustion at "heat sensitive points" on perennial allergic rhinitis," Zhongguo Zhen Jiu, vol. 28, no. 2, pp. 114 116, 2008 (Chinese).

[42] G. Li, "Therapeutic effect of Zhuang medicine medicated thread moxibustion on asthma of lung deficiency type," Zhongguo Zhen Jiu, vol. 25, pp. 181-183, 2005 (Chinese).

[43] X. Lai, Y. Li, Z. Fan, J. Zhang, and B. Liu, "An analysis of therapeutic effect of drug acupoint application in 209 cases of allergic asthma," Journal of Traditional Chinese Medicine, vol. 21, no. 2, pp. 122-126, 2001.

[44] X. Long, Q. Chang, and Q. Shou, "Clinical observation on 46 cases of infantile repeated respiratory tract infection treated by mild-moxibustion over acupoints on back," Journal of Traditional Chinese Medicine, vol. 21, no. 1, pp. 23-26, 2001.
[45] M. C. Cui and C. H. Li, "Clinical observation on drugseparated moxibustion at Shenque (CV 8) for treatment of infantile autumn diarrhea," Zhongguo Zhen Jiu, vol. 28, no. 3, pp. 194-196, 2008 (Chinese).

[46] X. Gao, J. Yuan, H. Li., and S. Ren, "Clinical research on acupuncture and moxibustion treatment of chronic atrophic gastritis," Journal of Traditional Chinese Medicine, vol. 27, no. 2, pp. 87-91, 2007.

[47] S. M. Wang, X. G. Li, L. Q. Zhang, Y. C. Xu, and Q. Li, "Clinical study on drug-seperated moxibustion at Shenque (CV 8) for treatment of ulcerative colitis," Zhongguo Zhen Jiu, vol. 26, pp. 97-99, 2006 (Chinese).

[48] H. Yu, M. Q. Zheng, and W. L. Wang, "Influence of moxibustion with small moxa-cone on the activity ptyalin and the contents of medium molecules in plasma to patients with diarrhea due to spleen-deficiency," Zhongguo Zhong Xi Yi Jie He Za Zhi, vol. 22, no. 1, pp. 65-66, 2002 (Chinese).

[49] X. Lin, J. Wu, and J. Du, "Study on effect of spine surgery on gastric function and its efficacy of relevant treatments," Zhongguo Zhong Xi Yi Jie He Za Zhi, vol. 20, no. 11, pp. 834836, 2000 (Chinese).

[50] H. G. Wu, L. B. Zhou, D. R. Shi et al., "Morphological study on colonic pathology ulcerative colitis treated by moxibustion," World Journal of Gastroenterology, vol. 6, no. 6, pp. 861-865, 2000.

[51] C. Y. Diao, Y. K. Yang, Y. H. Lu, Z. Wang, X. R. Yan, and Y. Wang, "Observation on clinical therapeutic effect of Yang's cotton moxibustion on neurodermatitis," Zhongguo Zhen Jiu, vol. 27, no. 3, pp. 176-178, 2007 (Chinese).

[52] J. X. Lao and Z. Y. Li, "Observation on therapeutic effect of acupuncture plus salt-partitioned moxibustion at Shenque (CV 8) in 60 cases of chloasma," Zhongguo Zhen Jiu, vol. 25, pp. 35-36, 2005 (Chinese).

[53] R. X. Chen, M. F. Kang, W. L. He, S. Y. Chen, and B. Zhang, "Moxibustion on heat-sensitive acupoints for treatment of myofascial pain syndrome: a multi-central randomized controlled trial," Zhongguo Zhen Jiu, vol. 28, no. 6, pp. 395-398, 2008 (Chinese).

[54] K. Sun, J. Yang, and D. K. Shen, "Clinical observation on treatment of primary knee osteoarthritis of liver and kidney deficiency type with Aconite cake-separated moxibustion," Zhongguo Zhen Jiu, vol. 28, pp. 87-90, 2008 (Chinese).

[55] J. W. Li, S. Y. Xiang, Z. Y. Ma et al., "Clinical observation on cake-separated mild-warm moxibustion for treatment of knee osteoarthritis," Zhongguo Zhen Jiu, vol. 28, no. 1, pp. 17-19, 2008 (Chinese).

[56] J. Jia, Q. Wang, T. Zhang, and J. Li, “Treatment of ankylosing spondylitis with medicated moxibustion plus salicylazosulfapyridine and methotrexate-a report of 30 cases," Journal of Traditional Chinese Medicine, vol. 26, no. 1, pp. 26-28, 2006.

[57] J. W. Li, J. M. Liu, Z. Y. Ma, Y. Y. Xiong, Y. B. Feng, S. Y. Xiang et al., "Clinical observation on treatment of rheumatoid arthritis with cake-separated mild moxibustion combined with Western medicine," Zhongguo Zhen Jiu, vol. 26, pp. 1924, 2006 (Chinese).

[58] Z. Xiaoxiang, "Jinger moxibustion for treatment of cervical vertigo-a report of 40 cases," Journal of Traditional Chinese Medicine, vol. 26, no. 1, pp. 17-18, 2006.

[59] L. Zhuang, "Twenty one cases of vertebral-artery-type cervical spondylosis treated with acupuncture and moxibustion," Journal of Traditional Chinese Medicine, vol. 20, no. 4, pp. 280281,2000 
[60] H. Sun, M. Y. Qiu, B. Q. Li et al., "Effect of moxibustion on quality of life in hemodialysis patients," Zhongguo Zhen Jiu, vol. 28, no. 5, pp. 321-324, 2008 (Chinese).

[61] S. P. Yun, W. S. Jung, S. U. Park et al., "Effects of moxibustion on the recovery of post-stroke urinary symptoms," American Journal of Chinese Medicine, vol. 35, no. 6, pp. 947-954, 2007.

[62] H. L. Liu and L. P. Wang, "Randomized controlled study on ginger-salt-partitioned moxibustion at shenque (CV 8) on urination disorders poststroke," Zhongguo Zhen Jiu, vol. 26, no. 9, pp. 621-624, 2006 (Chinese).

[63] W. L. Li, L. Liu, and L. H. Sun, "Analysis on therapeutic effect of substance-partitioned moxibustion at Guanyuan (CV 4) and shenque (CV 8) for treatment of primary dysmenorrhea of cold-damp type," Zhongguo Zhen Jiu, vol. 26, no. 7, pp. 481482, 2006 (Chinese).

[64] K. R. Shin, S. A. Kwak, J. B. Lee, and H. R. Yi, "The effectiveness of hand acupuncture and moxibustion in decreasing pain and "coldness" in Korea women who have had hysterectomy: a pilot study," Applied Nursing Research, vol. 19, no. 1, pp. 2230, 2006.

[65] S. M. Wang, X. G. Li, L. Q. Zhang, Y. C. Xu, and Q. $\mathrm{Li}$, "Clinical observation on medicine-separated moxibustion for treatment of primary dysmenorrhea and study on the mechanism," Zhongguo Zhen Jiu, vol. 25, pp. 773-775, 2005 (Chinese).

[66] L. Zhao, "Clinical observation on the therapeutic effects of heavy moxibustion plus point-injection in treatment of impotence," Journal of Traditional Chinese Medicine, vol. 24, no. 2, pp. 126-127, 2004.

[67] S. M. Peng, "Comparison of several methods for conversion of breech presentation," Zhongguo Yiyao Luntan, vol. 4, p. 30, 2006 (Chinese).

[68] F. Q. Yang, "Comparison of knee-chest position plus moxibustion on Zhiyin with knee-chest position for breech presentation," Sichuan Chinese Medicine, vol. 24, pp. 106-107, 2006 (Chinese).

[69] Y. Chen and L. W. Yang, "Moxibustion on Zhiyin plus raising buttocks in a lateral position for correction fetal presentation in 73 cases," Zhongyiyao Linchuang Zazhi, vol. 16, p. 333, 2004 (Chinese).

[70] Y. P. Lin, D. Q. Zhang, Y. Q. Hao, and X. W. Duan, "Combination of moxibustion at point Zhiyin and knee-chest position for correction of breech pregnancy in 63 cases," Zhongguo Zhenjiu, vol. 22, pp. 811-812, 2002 (Chinese).

[71] P. T. Dorsher, "The 2001 STRICTA recommendations for reporting acupuncture research: a review with implications for improving controlled clinical trial design," Journal of Alternative and Complementary Medicine, vol. 15, no. 2, pp. 147-151, 2009.

[72] B. Zhao, X. Wang, Z. Lin, R. Liu, and L. Lao, "A novel sham moxibustion device: a randomized, placebo-controlled trial," Complementary Therapies in Medicine, vol. 14, no. 1, pp. 5360, 2006.

[73] J. E. Park, C. H. Han, K. W. Kang, M. S. Shin, D. S. Oh, and S. M. Choi, "A sham moxibustion device and Masking test," Journal of Korean Oriental Medicine, vol. 13, pp. 93-100, 2007 (Korean).

[74] M. Sampson, N. J. Barrowman, D. Moher et al., "Should metaanalysts search Embase in addition to Medline?" Journal of Clinical Epidemiology, vol. 56, no. 10, pp. 943-955, 2003.
[75] H. MacPherson, A. White, M. Cummings, K. Jobst, K. Rose, and R. Niemtzow, "Standards for reporting interventions in controlled trials of acupuncture: the STRICTA recommendations," Complementary Therapies in Medicine, vol. 9, no. 4, pp. 246-249, 2001. 


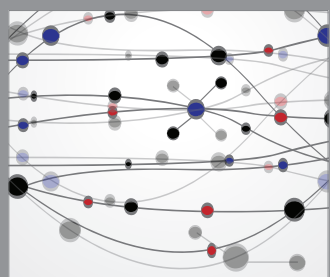

The Scientific World Journal
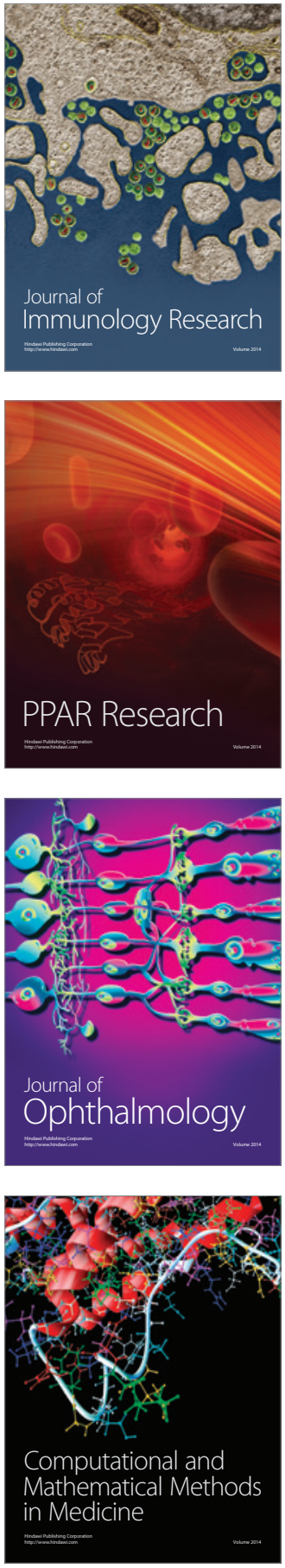

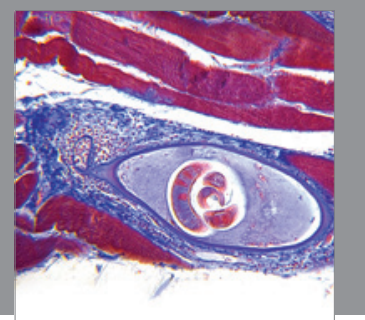

Gastroenterology

Research and Practice
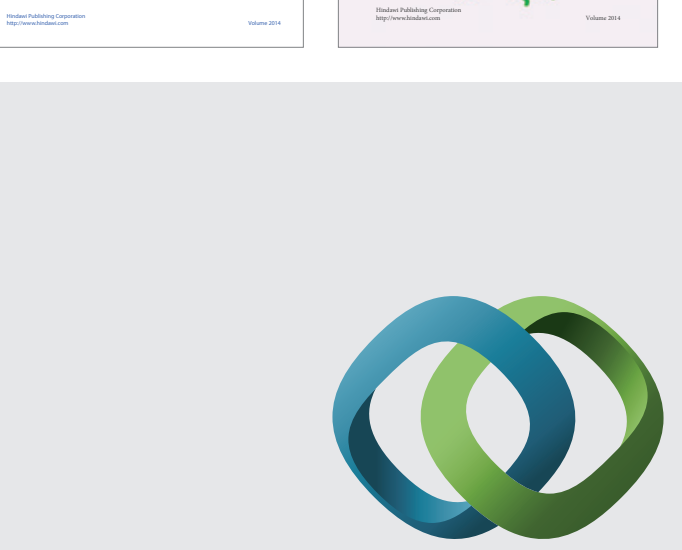

\section{Hindawi}

Submit your manuscripts at

http://www.hindawi.com
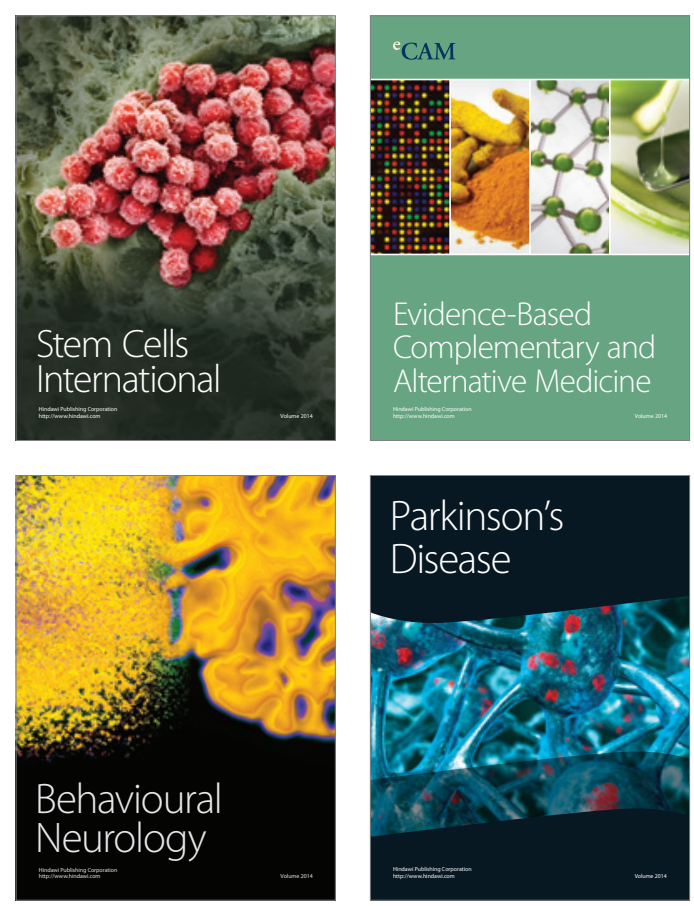

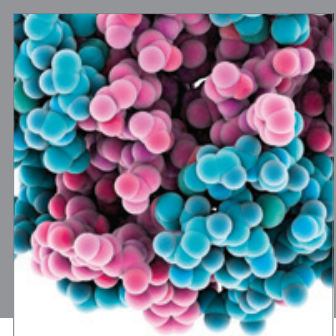

Journal of
Diabetes Research

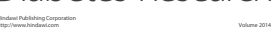

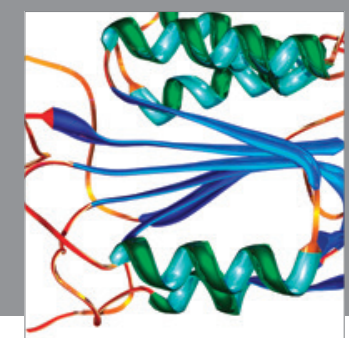

Disease Markers
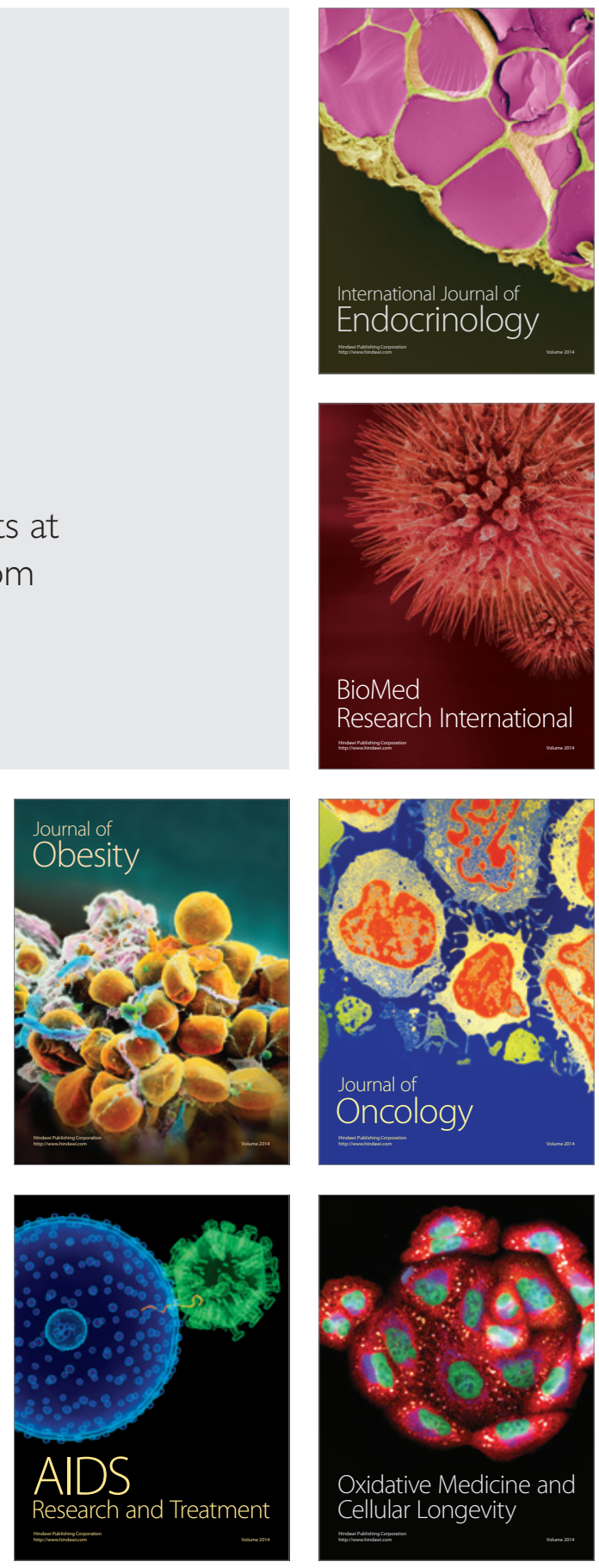